\title{
Rapid discrimination of klebsiella pneumoniae carbapenemase 2 - producing and non-producing klebsiella pneumoniae strains using near-infrared spectroscopy (NIRS) and multivariate analysis
}

\author{
Aline S. Marques ${ }^{a}$, Edgar P. Moraes ${ }^{a}$, Miguel A.A. Júnior ${ }^{b}$, Andrew D. Moura $^{\mathrm{b}}$, \\ Valter F.A. Neto ${ }^{\mathrm{b}}$, Renato M. Neto ${ }^{\mathrm{b}}$, Kássio M.G. Lima ${ }^{\mathrm{a}, *}$ \\ a Biological Chemistry and Chemometrics, Institute of Chemistry, Federal University of Rio Grande do Norte, Natal 59072-970, RN-Brazil \\ ${ }^{\mathrm{b}}$ Laboratory of Mycobateria, Department of Microbiology and Parasitology, Federal University of Rio Grande do Norte, Natal 59072-970, RN-Brazil
}

\section{A R T I C L E I N F O}

\section{Article history:}

Received 8 September 2014

Received in revised form

1 November 2014

Accepted 3 November 2014

Available online 12 November 2014

Keywords:

Carbapenemase

Klebsiella pneumoniae

Near infrared spectroscopy

SPA-LDA

GA-LDA

\begin{abstract}
A B S T R A C T
Klebsiella pneumoniae Carbapenemase (KPC-2)-producing and non-producing Klebsiella pneumoniae (KP) have rapidly disseminated worldwide, challenging the diagnostics of Gram-negative infections. We evaluate the potential of a novel non-destructive and rapid method based on Near-Infrared Spectroscopic (NIRS) and multivariate analysis for distinguishing KPC-2 - producing and non-producing KP. Thirty-nine NIRS spectra (24 KPC-2-producing KP, 15 KPC-2 non-producing KP) were acquired; different pre-processing methods such as baseline correction, derivative and Savitzky-Golay smoothing were performed. A spectral region fingerprint was achieved after using genetic algorithm-linear discriminant analysis (GA-LDA) and successive projection algorithm (SPA-LDA) algorithms for variable selection. The variables selected were then used for discriminating the microorganisms.Accuracy test results including sensitivity and specificity were determined. Sensitivity in KPC-2 producing and non-producing KP categories was $66.7 \%$ and $75 \%$, respectively, using a SPA-LDA model with 66 wavenumbers. The resulting GA-LDA model successfully classified both microorganisms with respect to their "fingerprints" using only 39 wavelengths. Sensitivity in KPC-2 producing category was moderate( $\approx 66.7 \%)$ using a GA-LDA model. However, sensitivity in KPC-2 non-producing category using GA-LDA accurately predicted the correct class (with 100\% accuracy). As100\% accuracy was achieved, this novel approach identifies potential biochemical markers that may have a relation with microbial functional roles and means of rapid identification of KPC-2 producing and non-producing KP strains.
\end{abstract}

(C) 2014 Elsevier B.V. All rights reserved.

\section{Introduction}

Gram-negative bacilli (GNB) of clinical importance can be divided into two major groups. Glucose-fermenting, oxidasenegative, and catalase-positive members constitute one group, called Enterobacteriaceae [1]. Resistance to carbapenem among members of the Enterobacteriaceae family has become a major health care concern worldwide [2]. Klebsiella pneumoniae carbapenemase (KPC)-producing bacteria are an emerging group of highly drug-resistant bacteria causing infections associated with significant morbidity and mortality [3]. Among the most common clinical syndromes associated with KPC-producing bacteria presence are: pneumonia, urinary tract infections and manifestations of wounds, bacteremia, chronic atrophic rhinitis, arthritis, dysentery, meningitis, and sepsis in children, particularly those acquired

\footnotetext{
* Corresponding author. Tel.: +55 843342 2323; fax: +55 8332119224 .

E-mail address: kassiolima@gmail.com (K.M.G. Lima).
}

in hospital [4]. The genes for the 10 known KPC variants (KPC 2-11) are carried on large plasmids [5]. Detection of KPC-2 producing bacteria may be a challenge for clinical laboratories because in this study it was associated with positive extended-spectrum $\beta$-lactamase (ESBL) confirmation tests (clavulanate-potentiated activities of ceftriaxone, ceftazidime, cefepime, and aztreonam) [6].

Traditionally, the tests for detection of KPC-type producing bacteria and other microorganisms are combined in a series of solid and/or liquid media which are inoculated with bacteria and interpreted/analyzed after a certain incubation period. The classical approaches are based on agar diffusion methods [7], broth microdilution (BMD) [8], modified Hodge test [9], polymerase chain reaction (PCR) - based assays [10], among others. Although these assays have achieved good sensitivity and specificity with favorable positive and negative predictive values for these microorganisms, they often require multiple steps with additional time needed for the clarification/discernment of species and/or detection of antimicrobial resistance. Moreover, slow multistep culturebased assays are time consuming, labor-intensive and require 
skilled clinical microbiologists working in the lab. There is a need for a quicker, non-destructive, sensitive, and specific means of detecting and differentiating KPC-type producing bacteria strains.

Over the past decades, advanced molecular techniques in diagnostic microbiology have been revolutionizing the practice of clinical microbiology [11]. In particular, Near-Infrared Spectroscopy (NIRS) provides the ability to quickly detect analytes and has been explored as an interesting alternative tool for the identification of microbial species and subspecies [12-15]. Its sensitivity to the $\mathrm{CH}, \mathrm{NH}$, and $\mathrm{OH}$ absorptions related to microbial components, its speedy response time, the simplicity of sample preparation involved, the fact that the measurement is non-destructive, and its low instrumentation cost have fixed its position alongside other spectroscopies, including ultraviolet, visible, mid-infrared, Raman, and others.

Marques et al. [12] (2013) described the usefulness of NIRS in the identification and classification of Escherichia coli and Salmonella enteritidis from commercial fruit pulp (pineapple). The authors obtained good performance achieving prediction ability of $87.5 \%$ for E. coli and $88.3 \%$ for S. enteritidis, respectively. Rodriguez-Saona et al. [13] investigated the feasibility of NIRS in microbiology and the development of methodology for the quick identification of bacterial strains such as Escherichia coli HB101, non-virulent strain of Escherichia coli ATCC 43888, Escherichia coli ATCC 1224, Pseudomonas aeruginosa, Bacillus amyloliquifaciens, Bacillus cereus, and Listeria innocua. The use of NIRS spectral information and multivariate techniques in this study showed potential for the identification and subtyping of different bacterial species. Arnold et al. [14] used NIRS to monitor a submerged filamentous bacterial (Streptomyces fradiae) bioprocess. The present work is original/new in fully reporting how NIRS can be used to simultaneously measure the concentration of key analytes atline in an antibiotic production process involving a filamentous bacterium, and in detailing the actual modeling process and subsequent critical assessment of model quality and performance.

However, the method of data analysis is a critical aspect of any diagnostic assay, particularly for NIR. The major difficulties in the analysis of microbial species and subspecies are the weakness of the NIR signals from strains components and the complexity of overlapping bands. To overcome these difficulties, many chemometric algorithms have been applied to NIR data such as principal component analysis (PCA) for initial data reduction and exploratory data analysis [16], hierarchical cluster analysis (HCA) for analyzing groups in a set of data on the basis of spectral similarities [17], and linear discriminant analysis (LDA) for classifying unknown samples into predetermined groups [18]. Further, as part of the computational methodology, variable selection methods such as successive projections algorithm (SPA) [19] in conjunction with LDA and genetic algorithm (GA) [20] improve the model performance compared with the full spectrum model. These algorithms eliminate potential interferents and variables that generate a lower signal/noise ratio.

Although these studies have shown that NIR together with chemometrics analysis have been explored as alternative tools for the identification of microbial species, little research has been directed toward the use of NIRS and variable selection to be used in pathogenic microbiology studies. Herein, we have attempted to evaluate the potential of a quicker method for identification of KPC-2-producing and non-producing Klebsiella pneumoniae strains. For this, the present article set out to determine whether biochemical intra-individual differences or "fingerprint" features between KPC-2-producing and non-producing bacteria could be identified using NIR spectroscopy with subsequent variable selection methods. We employed SPA and GA to select an appropriate subset of wavenumbers for LDA. This approach can lead to more selective and specific microbial detection for medically relevant microorganisms by vibrational spectroscopy. Nevertheless, KPC-2producing and non-producing Klebsiella pneumoniae was never discriminated by NIRS using wavelength selection to elucidate the altered biochemical-microbial fingerprint.

\section{Material and methods}

\subsection{Bacteria strains}

Specimens of Enterobacteriaceae family from different biological sites were recruited from three health centers in the city of Natal / Rio Grande do Norte, Brazil from April 2012 to August 2013. The specimens were cultured on blood agar (HIMEDIA), Mac Conkey Agar (HIMEDIA) and Brain Heart Infusion Broth (BHI/ HIMEDIA) followed by inoculation in conventional atmosphere at $35^{\circ} \mathrm{C}$ for a period of $24 \mathrm{~h}$. The phenotypic identification was confirmed by the Vitek automation system (BioMérieux Vitek, St. Louis). The antimicrobial susceptibility testing, as well as confirming the production of ESBL and KPC-producing tests were determined by the disk - agar diffusion (Kirby - Bauer), as recommended by the CLSI 2013.

\subsection{Analysis of molecular pattern multi-resistance Enterobacteriaceae}

Enterobacteriaceae with phenotypic standard for ESBL [21] and KPC genotypic analysis of the genes bla TEM, bla SHV, bla CTX -M and bla KPC were sent. For extraction and purification of total DNA, 250 QIAamp DNA Mini Kit (Cat. no. 51306) from Qiagen kit was used. The types of ESBL and KPC were determined through custom protocol to Polymerase Chain Reaction (PCR) using specific primers. The primer pair 5'-ATTCTTGAAGACGAAAGGGC-3' (forward) and 5'-ACGCTCAGTGGAACGAAAAC-3' (reverse) was used for amplification of a sequence of 1150 base pairs (pb) from the TEM family [22]. For the SHV family, the primer pair 5'-GGGTTATTCTTATTTGTCGC-3' (forward) and 5'-TTAGCGTTGCCAGTGCTC-3' (reverse) (947pb) was used, for the CTX-M family, 5'-TTTGCGATGTGCAGTACCAGTAA-3' (forward) and 5'- CGATATCGTTGGTGGTGCCATA-3 ' (reverse) (544pb) [23] and KPC2,5- TGTCACTGTATCGCCGTC-3 (forward) and 5- CTCAGTGCTCT ACAGAAAAACC-3 (reverse) (1100pb) [24] were used. The PCR was performed using an appropriate protocol in a final volume of $25 \mu \mathrm{L}$ containing $1 \mu \mathrm{L}$ of DNA, $16.25 \mu \mathrm{L}$ of nuclease-free water, $2.5 \mu \mathrm{L}$ of Taq buffer $10 \mathrm{X}, 2 \mu \mathrm{L}$ of deoxynucleotide mixture, $0.75 \mu \mathrm{L}$ of $\mathrm{MgCl}_{2}$ $\left(50 \mathrm{mmol} \mathrm{L}^{-1}\right), 1 \mu \mathrm{L}(10 \mathrm{pmol})$ of each "primer" and $0.3 \mu \mathrm{L}$ of Taq DNA polymerase ( $5 \mathrm{U} \mu \mathrm{L}^{-1}$, Ludwig, Alvorada /RS/Brazil). For the amplification conditions of the bla gene CTX-M, bla SHV, bla TEM and bla KPC were used at $94{ }^{\circ} \mathrm{C}$ pre-denaturation, 30 cycles of $1 \mathrm{~min}$ at $94{ }^{\circ} \mathrm{C}$ (denaturation), $2 \mathrm{~min}$ at $57.2^{\circ} \mathrm{C}$ for annealing, $2 \mathrm{~min} 72{ }^{\circ} \mathrm{C}$ for extension, succeeded by a final extension of $8 \mathrm{~min}$ at $72^{\circ} \mathrm{C}$. After the PCR reaction, the visualization of the amplified fragments was performed by agarose gel electrophoresis (Ludwig) $1 \%$ in $1 \mathrm{X}$ TAE (Tris-acetate).

\subsubsection{NIR spectroscopy}

Each NIR spectra $\left(8 \mathrm{~cm}^{-1}\right.$ spectral resolution, co-added for 32 scans and in triplicate) were directly acquired, in reflectance mode on a miniature scanning Fourier-Transform spectrometer from ARCspectro ANIR (Neuchâtel, Switzerland), which is based on a lamellar grating interferometer $(35 \mathrm{~mm} \times 35 \mathrm{~mm} \times 65 \mathrm{~mm})$ and uses a micro-mechanical actuator. The portable NIR device uses an InGaAs photodiode (900 $\mathrm{nm}$ to $2600 \mathrm{~nm}$ ) and the reflected light was directed to the spectrometer through a bundle of optical fibers (model R600-7-VIS-125F, Ocean Optics, USA) linked to the probe end and the data acquisition and analyses were carried out by ARCspectro ANIR 1.64 software. Absorbance spectra of DNA 
samples were obtained against the spectrum of reflectance standard (Labsphere, 8750) used as background. A disposable syringe $(1 \mathrm{~mL})$ was used to place the samples $(0.1 \mathrm{~mL})$ on an aluminumplated backing plate $(0.1 \mathrm{~mm}$ sample thickness). The transflectance probe was positioned on the sample surface. The transflectance probe was washed with ethanol (70\% v/v) and dried using tissue paper after each sample. Cleanliness of the transflectance probe was verified by collecting an absorbance spectrum of the probe using the most recently collected background as a reference. Spectral measurements were done in an acclimatized room under controlled temperature of $22{ }^{\circ} \mathrm{C}, 60 \%$ relative air humidity, and samples were allowed to equilibrate to this temperature before analysis. Sample positioning, data collection, and storage took less than one minute per sample. Ninety spectra (45 KPC-2-producing Klebsiella pneumoniae and 45 KPC-2-non-producing Klebsiella pneumoniae) were randomized. Fig. 1 shows the experimental arrangement for sampling discrimination of KPC-2-producing and non-producing Klebsiella pneumonia using NIR spectroscopy.

\subsection{Chemometrics methods: PCA, SPA-LDA and GA-LDA}

For microbial identification, two basic approaches can be applied in the chemometric techniques: unsupervised and supervised techniques. The objective of unsupervised methods, also called exploratory methods, is to depict the spectral data, without prior knowledge about the microorganism studied. Principal component analysis (PCA) [25] is a well-known unsupervised way to reduce the number of variables, in which the spectral matrix $\mathrm{X}$ is decomposed as:

$X=T \mathbf{P}^{\mathbf{T}}+E$,

where $\mathrm{X}$ is the $I \times J$ data matrix, $\mathrm{T}$ is the $I \times A$ matrix of score vectors, the score vectors $t_{a}$ are orthogonal (i.e., $T^{\mathrm{T}} T=\operatorname{diag}\left(\lambda_{a}\right)$ and $\lambda_{a}$ are eigenvalues of the matrix $\mathrm{X}^{\mathrm{T}} \mathrm{X}$ ), $\mathrm{P}$ is the $J \times A$ matrix of loadings vectors, superscript ${ }^{\mathrm{T}}$, as usual, indicates the transpose of a matrix, $\mathrm{E}$ is the $I x J$ residual matrix, $I$ is the number of objects, $J$ is the number of variables, and $A$ is the number of components calculated. In other words, PCA is often used for reducing the dimensionality of the data without decreasing their variance, and each spectrum is then compared to the others in order to make homogeneous groups.

The second approach is based on supervised techniques coupled with variable selection methods such as genetic algorithm-linear discriminant analysis (GA-LDA) and successive projection algorithm (SPA-LDA). In this report, LDA will refer to the canonical discriminant procedure developed by Fisher in 1936 [26] and designed to maximize between-groups variability relative to a measure of pooled within-groups variability. The variables created through LDA (factors) are linear combinations of the wavenumberabsorbance intensity values [27]. Thus, the use of LDA for identification or classification of spectral data generally requires appropriate variable selection procedures [28]. In the present study, the validation samples were performed in SPA and GA to select the best optimum number of variables by minimizing a cost function calculated as:

$G=\frac{1}{N_{V}} \sum_{n=1}^{N_{V}} g_{n}$

where $g_{n}$ is defined as

$g_{n}=\frac{r^{2}\left(x_{n}, m_{I(n)}\right)}{\min _{I(m) \neq l(n)} r^{2}\left(x_{n}, m_{I(m)}\right)}$

where $I(n)$ is the index of the true class for the nth validation object $x_{n}$.

For this study, LDA scores, loading, and discriminant function (DF) values were derived for the biochemical-bacteria fingerprint region. The first LDA factor (LD1) was used to visualize the alterations of the bacteria sample in 1-dimensional (D) score plots that represented the main biochemical alterations.

\subsubsection{Software}

Data loading, pre-processing (mean-centering, Savitzky-Golay smoothing with different windows (3, 5, 7 and 15), first polynomial order, derivatization of first and second derivatives) and PCA were implemented in a MATLAB $\Re$ version 7.10 environment (Math-Works, Natick, USA) with the PLS-toolbox version 7.5.2 (Eigenvector Research, Inc., Wenatchee, WA). The KS, GA-LDA and SPA-LDA classification routines were implemented in

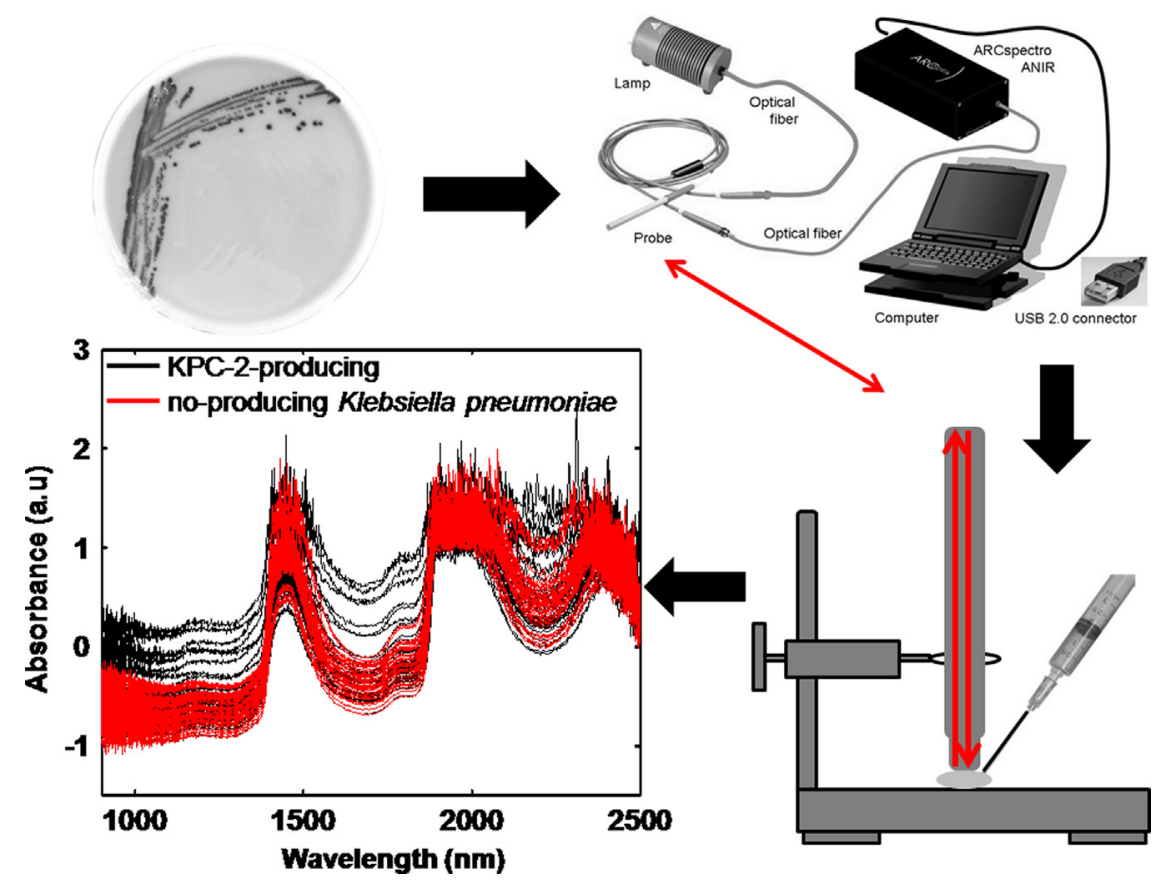

Fig. 1. Experimental arrangement for sampling discrimination of KPC-2-producing and no-producing Klebsiella pneumoniae using NIR spectroscopy. 
Matlab ${ }_{R}$ 7.10. For SPA-LDA and GA-LDA models, each class was treated separately. The Kennard Stone (KS) algorithm [29] was employed to select the samples which would compose the training ( $60 \%$ of samples), validation ( $20 \%$ of samples) and test $(20 \%$ of samples) sets to the NIR spectra. Mean centering was applied to all spectra before performing variable subset selection and calibration. The training and validation samples were used in the modeling procedures (including variable selection for LDA), whereas the test samples were only used in the final evaluation and comparison of the classification models.

For the GA routine, the initial population was 120 individuals with 60 generations each. The mutation and reproduction probabilities were kept constant at 10 and 60\%, respectively. The best solution (in terms of the fitness value) resulting from the three realizations of the GA was kept. The final results (SPA-LDA and GALDA models) were expressed in terms of classification rates for the validation set.

Receiver-operating characteristic (ROC) analysis is recommended standard practice for test evaluation studies for nonbinary tests [30]. For this study, measures of test accuracy, such as sensitivity (probability that a test result will be positive when the disease is present), specificity (probability that a test result will be negative when the disease is not present) were calculated as important quality standards in test evaluation.

\section{Results and discussion}

\subsection{NIR spectra}

As can be seen in Fig. 1, NIR spectra acquired from two classes (KPC-2-producing and non-producing Klebsiella pneumoniae) present a consistent baseline offset and bias. Although these are quite common features in NIR spectra acquired by diffuse reflectance techniques [31], some pre-treatments need to be performed. Among the pre-processing techniques tested, the one showing better separation of the classes employing PCA, SPA-LDA and GALDA was the combination of Savitzky-Golay first derivative (15 points window, first degree polynomial) and Savitzky-Golay smoothing (15 points window). In all cases, mean-centering was effectively applied to calculate the average spectrum of the data set and subtract the average from each spectrum. A first-derivative transformation makes unique spectral features of the different bacteria strains more prominent. In addition, first-derivative transformation is often applied to process spectral data since it separates overlapping absorption bands, removes baseline shifts and increases apparent spectral resolution. These effects are shown in Fig. 2.

As seen in Fig. 2, the consistent baseline offset and bias after processing were corrected and now it is possible to assign some

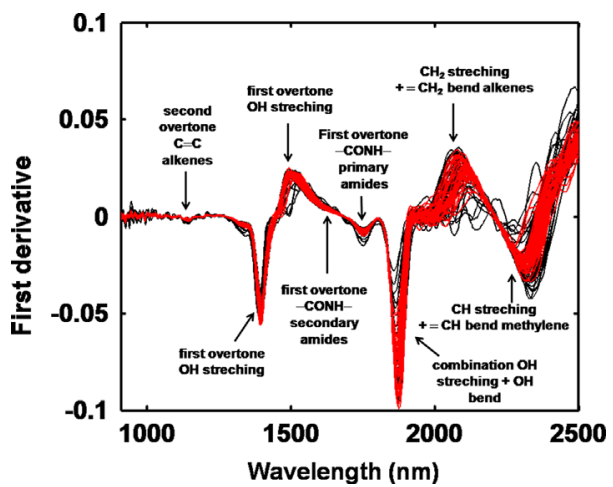

Fig. 2. NIR spectra with first derivative of the Savitzky-Golay using a window of fifteen points. (-) KPC-2-producing; ( - ) no-producing Klebsiella pneumoniae. overtones and combination bands evidenced in the spectrum, including the following: a weak band at approximately $1140 \mathrm{~nm}$ was influenced by absorption exerted by the second overtonerelated alkenes; absorption peaks were recorded at approximately 1398 and 1485, both associated with the first overtone $\mathrm{OH}$ stretching (water); a band at approximately $1632 \mathrm{nn}$ is associated with the first overtone - $\mathrm{CONH}$ - secondary amides, a probable biochemical signature from DNA/RNA (cytosine, guanine and uracil), while at $1756 \mathrm{~nm}$ there was influence from absorption exerted by the first overtone - $\mathrm{CONH}$ - primary amides; a strong band at approximately $1900 \mathrm{~nm}$ was related to $\mathrm{OH}$ combination (stretching and bend modes); and the regions from 2110 to $2205 \mathrm{~nm}$ and from 2250 to $2300 \mathrm{~nm}$ were assigned to alkenes $\left(\mathrm{CH}_{2}\right.$ stretching and bend) and methylene $(\mathrm{CH}$ stretching and bend), respectively. Tentative assignments to KPC-2-producing and non-producing Klebsiellapneumoniae were based on systematic comparison of these major regions with the band known [32].

\subsection{Principal component analysis}

After processing, the range of $900-2600 \mathrm{~nm}$ was submitted to PCA analysis. The PCA model was built from the calibration set using 2 PC explaining together $65.34 \%$ of the variance in the data after applying the pre-processing (Savitzky-Golay smoothing and first derivative). As can be seen in Fig. 3a, the classes are overlapped. This weak separation was obtained without using the information that the samples belong to two different groups and is indicative of features of/with spectral differences. The major loadings (Fig. 3b) are in the $1400-1410 \mathrm{~nm}$ (first overtone $\mathrm{OH}$ stretching) region with other contributing regions at $1890-1910 \mathrm{~nm}(\mathrm{OH}$ combination) and 2150-2400 nm (alkenes and methylene bands).
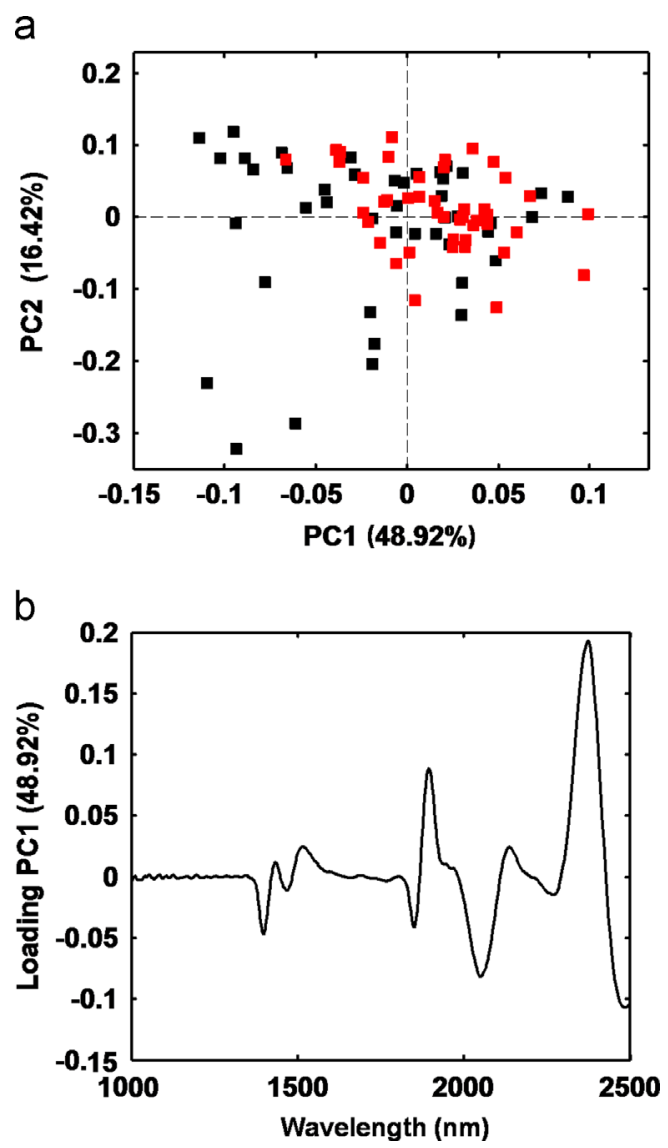

Fig. 3. PCA results: (a) score plot of PC1 versus PC2 (KPC-2-producing: -, noproducing Klebsiella pneumoniae: and (b) loading plot (PC1, 48.92\%). 
a

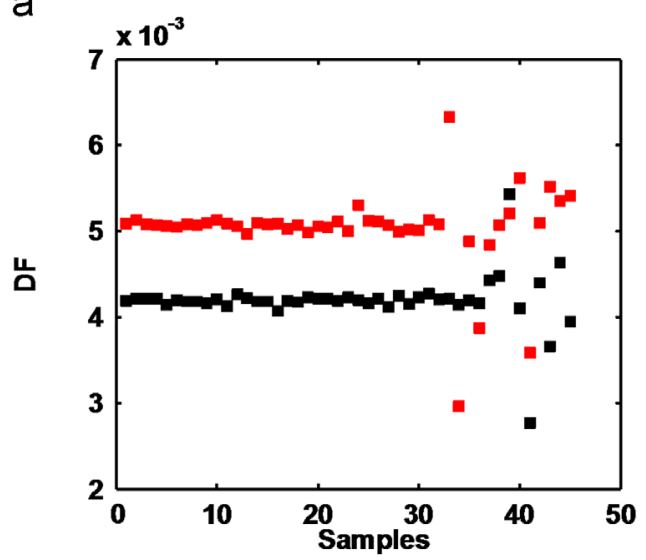

b

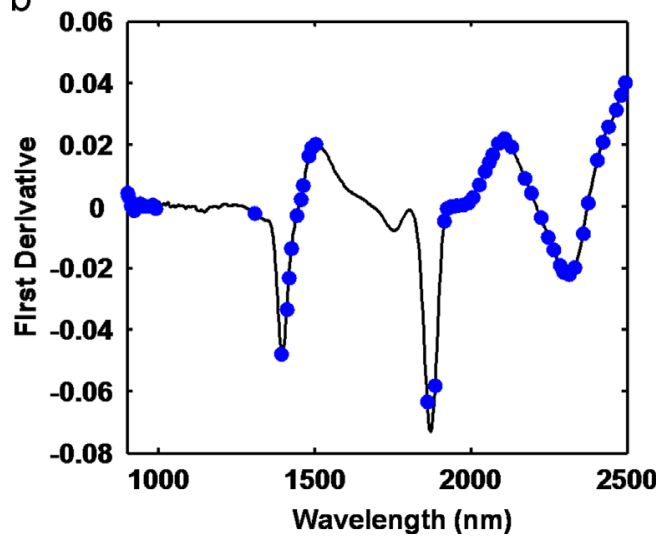

Fig. 4. (a) DF1 $\times$ samples discriminant function values calculated by using the variables selected by SPA-LDA of the data set (KPC-2-producing: - , no-producing Klebsiella pneumoniae: a; (b) the blue full circles indicate the position in the spectra of the 66 wavelengths variables by using the variables selected by SPA-LDA of the data set.

\subsection{SPA-LDA and GA-LDA results}

SPA was applied to the data set (KPC-2-producing versus nonproducing Klebsiella pneumoniae) and resulted in the selection of 66 variables, namely 900, 903, 911, 921, 930, 934, 942, 949, 963, $981,990,1308,1393,1410,1417,1425,1443,1455,1463,1481,1489$, 1502, 1860, 1885, 1914, 1923, 1937, 1943, 1956, 1974, 1990, 2006, 2025, 2045, 2059, 2069, 2086, 2107, 2129, 2173, 2192, 2224, 2248, 2264, 2285, 2297, 2314, 2332, 2358, 2376, 2403, 2422, 2441, 2465, 2480, 2494, 2520, 2530, 2540, 2556, 2561, 2577, 2587, 2593, 2598 and $2600 \mathrm{~nm}$. Using the 66 selected wavelengths, the Fisher scores were obtained and there was also good segregation from each class. Fig. 4a shows the Fisher scores and Fig. 4b indicates the wavelength selected by SPA-LDA. Upon examination of the selected wavenumbers following SPA-LDA (Fig. 4b) indicate that the main biochemical alterations were on $\mathrm{CH}_{2}$ stretching $+\mathrm{CH}_{2}$ bending of vinyl alkenes $(2192 \mathrm{~nm})$ and $\mathrm{CH}$ stretching $+\mathrm{C}=\mathrm{O}$ stretching of CHO aldehydes $(2224 \mathrm{~nm})$.The SPA-LDA model achieved a sensitivity of $66.7 \%$ and $75.0 \%$, respectively, for KPC2-producing versus non-producing $\mathrm{KP}$.

GA-LDA model for comparison achieved an improvement in segregation between KPC-2-producing versus non-producing KP. The GA resulted in the selection of 39 wavenumbers (of 909 available); these were 914, 927, 939, 958, 973, 993, 1065, 1075, 1128, 1134, 1144, 1157, 1178, 1190, 1200, 1270, 1318, 1322, 1341, $1342,1372,1376,1391,1438,1458,1458,1576,1592,1596,1600$, $1606,1824,1943,1953,1993,2340,2367,2399,2450$ and $2545 \mathrm{~nm}$. Using the 39 selected wavelengths (Fig. 5a),the Fisher scores for all a
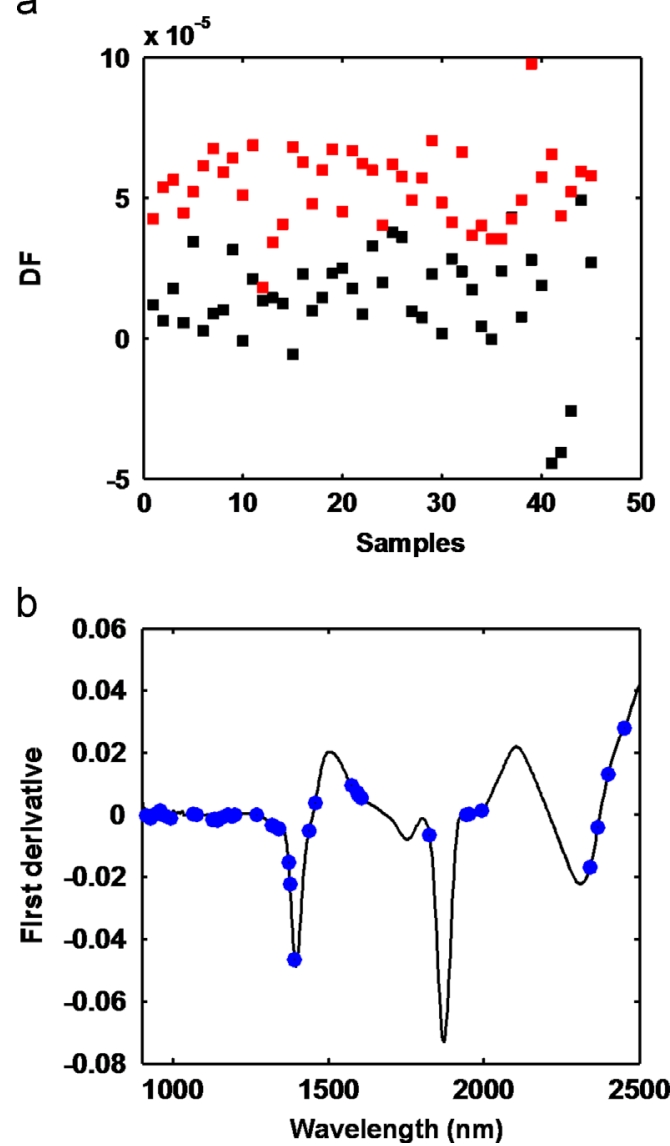

Fig. 5. (a) DF $1 \times$ samples discriminant function values calculated by using the variables selected by GA-LDA of the data set (KPC-2-producing: -, no-producing Klebsiella pneumoniae: $\square$; (b) the blue full circles indicate the position in the spectra of the 39 wavelengths variables by using the variables selected by GA-LDA of the data set.

the samples of the data set (Fig. 5b) were obtained whose minimum point cost function was achieved with 39 wavenumbers. As can be seen, there was again an excellent separation from each category. The accuracy of GA-LDA for KPC-2-producing was $66.7 \%$ for sensitivity. For non-producing KP, GA-LDA model achieved a sensibility of $100.0 \%$. Several selected wavenumbers appear to be of particular interest, namely the variables at 1318 and $2340 \mathrm{~nm}$, representing the $\mathrm{CH}$ stretching and bending of methylene and $\mathrm{NH}$ stretching and bending of amide primary, respectively.

\section{Conclusion}

As was demonstrated, NIR spectroscopy facilitates the derivation of an integrated biochemical-bacteria fingerprint based on the NIR absorbing properties of the constituent chemical vibrations. The potential of NIR spectroscopy to discriminate KPC-2- producing and non-producing bacteria has been examined in this work. The study clearly demonstrates that different phenotypes of bacteria can be clearly segregated using NIR spectroscopy with subsequent PCA, SPA-LDA and GA-LDA algorithms. These findings have at least two important implications. First, NIRS and computational analysis can be applied as a novel approach to investigate these microbial pathogens because the timely and accurate detection/identification is critical for patient treatment decisions and outcomes for millions of patients each year. Second, with the miniaturization of instruments for field measurements employing light emission diode (LED) that emit radiation at wavelengths 
previously selected (e.g., NIR), this approach may be used in analysis or discriminatory simultaneous determinations based on multivariate analysis [33].

\section{Acknowledgments}

The authors would like to acknowledge the financial support from the CAPES for a fellowship for Marques, A.S, the Graduate Program in Chemistry (PPGQ) of UFRN and mycobacteria, malaria and toxoplasmosis Laboratories. The authors thank Dra. Marise Dutra Asensi (Laboratório de Pesquisa em Infecção Hospitalar/ LAPIH-Fio Cruz/RJ) for providing the bacterial strains control. The work was funded by grants from CNPq/Capes project (Grant 070/ 2012) and FAPERN (Grant 005/2012).

\section{References}

[1] D.L. Paterson, Am. J. Med. 119 (2006) S20-S28.

[2] P. Nordmann, G. Cuzon, T. Naas, Lancet Infect. Dis. 9 (2009) 228-236.

[3] C.P. Thomas, L.S.P. Moore, N. Elamin, M. Doumith, J. Zhang, S. Maharjan, M. Warnerc, C. Perryc, J.F. Turtonc, C. Johnstonea, A. Jepsona, N.D.C. Duncana, A.H. Holmesa, Antimicrob. Agents 42 (2013) 531-536.

[4] I. Pena, J.J. Picazo, C. Rodríguez-Avial, I. Rodríguez-Avial, Int. J. Antimicrob. Agents 43 (5) (2014) 460-464.

[5] F. Perez, A. Endimiani, A.J. Ray, B.K. Decker, C.J. Wallace, K.M. Hujer, D.J. Ecker, M.D. Adams, P. Toltzis, M.J. Dul, A. Windau, S. Bajaksouzian, M.R. Jacobs, R.A. Salata, R.A. Bonomo, J. Antimicrob. Chemoth. 65 (2010) 1807-1818.

[6] Clinical Laboratory Standards Institute. Performance Standards for Antimicrobial Susceptibility Testing: Twenty-third Informational Supplement M100S23, CLSI, Wayne, PA, USA, 2013.

[7] S. Bratu, D. Landman, R. Haag, R. Recco, A. Ermo, M. Alam, J. Quale., Arch. Intern. Med. 165 (2005) 1430-1435.

[8] J.H. Jorgensen, J.C. Lee, Microdilution Technique for Antimicrobial Susceptibility Testing of Haemophilus influenzae 8 (1975) 610-612.

[9] K. Lee, Y. Chong, H.B. Shin, Y.A. Kim, D. Yong, J.H. Yum, Clin. Microbiol. Infect. 7 (2001) 88-91.

[10] J.M. Cole, A.N. Schuetz, C.E. Hill, F.S. Nolte, J. Clin. Microbiol. 47 (2009) 322-326.
[11] K. Maquelin, C. Kirschner, L.-P. Choo-Smith, N. van den Braak, H.P. Endtz, D. Naumann, G.J. Puppels, J. Microbiol. Methods 51 (2002) 255-271.

[12] A.S. Marques, J.T.N. Nicácio, T.A. Cidral, M.C.N. de Melo, K.M.G. de Lima, J. Microbiol. Methods 93 (2013) 90-94.

[13] L.E. Rodriguez-Saona, F.M. Khambaty, F.S. Fry, E.M. Calvey, J. Agric. Food Chem. 49 (2001) 574-579.

[14] S. Arnold, J. Crowley, S. Vaidyanathan, L. Matheson, P. Mohan, J. Hall, L.M. Harvey, B. McNeil, Enzyme Microb. Technol. 27 (2000) 691-697.

[15] D. Alexandrakis, G. Downey, A.G.M. Scannel, J. Agric. Food Chem. 56 (2008) 3431-3437.

[16] Y. He, X. Li, X. Deng, J. Food Eng. 79 (2007) 1238-1242.

[17] M.T. Bona, J.M. Andrés, Talanta 72 (2007) 1423-1431.

[18] H. Yang, J. Irudayaraj, M. Paradkar, Food Chem. 93 (2005) 25-32.

[19] M.J.C. Pontes, R.K.H. Galvão, M.C.U. Araújo, P.N.T. Moreira, O.D.P. Neto, G.E. José, T.C.B. Saldanha, Chemometr. Intell. Lab. Syst 78 (2005) 11-18.

[20] H.S. Tapp, M. Defernez, E.K. Kemsley, J. Agric. Food Chem. 51 (2003) 6110-6115.

[21] V. Jarlier, M.H. Nicolas, G. Fournier, A. Philippon, Rev. Infect. Dis. 10 (1988) $867-878$.

[22] C.D. Steward, J.K. Rasheed, K. Susannah, J.W. Biddle, P.M. Raney, G.J. Anderson, P.P. Williams, K.L. Brittain, A. Oliver, J.E. McGowan Jr., F.C. Tenover, J. Clin. Microbiol. 39 (2001) 2864-2872.

[23] J.D.D. Pitout, D.L. Church, D.B. Gregson, B.L. Chow, M. Mccracken, M.R. Mulvey, K.B. Laupland, Antimicrob. Agents Chemother. 51 (2007) 1281-1286.

[24] H. Yigit, A.M. Queenan, G.J. Anderson, A. Domenech-sanchez, J.W. Biddle, C.D. Steward, S. Alberti, K. Bush, F.C. Tenover, Antimicrob. Agents Chemoth. 45 (2001) 1151-1161.

[25] B.M. Wise, N.L. Ricker, D.F. Veltkamp, B.R. Kowalski, Process Contr. Qual 1 (1990) 41-51.

[26] R.A. Fisher, Ann Eugen 7 (1936) 179-188.

[27] F.L. Martin, M.J. German, E. Wit, T. Fearn, N. Ragavan, H.M. Pollock, J. Comput, Biol. 14 (2007) 1176-1184.

[28] A.S. Marques, M.C.N. de Melo, T.A. Cidral, K.M.G. de Lima, J. Microbiol. Methods 98 (2013) 26-30.

[29] R.K.H. Galvão, M.C.U. Araujo, G.E. José, M.J.C. Pontes, E.C. Silva, T.C.B. Saldanha, Talanta 67 (2005) 736-740.

[30] K.T. Cheung, J. Trevisan, J.G. Kelly, K.M. Ashton, H.F. Stringfellow, S.E. Taylor, M.N. Singh, P.L. Martin-Hirsch, F.L. Martin, Analyst 136 (2011) 2047-2055.

[31] E.W. Ciurczak, Principles of near-infrared spectroscopy, in: D.A. Burns, E.W. Ciurczak (Eds.), Handbook of Near-Infrared Analysis, Marcel Dekker, New York, 2001, pp. 7-18.

[32] H.W. Siesler, Y. Ozaki, K. Satoshi, H.M. Heise, Near-Infrared Spectroscopy: Principles, Instruments, Applications, Wiley-VCH, Weinheim, 2002.

[33] K.M.G. de Lima, Microchem. J. 103 (2012) 62-67. 